\title{
Enseñar Diseño: La emergencia de la teoría
}

\section{Cravino, Ana}

Resumen:

El objetivo de este trabajo es

reflexionar sobre algunas

aseveraciones manifestadas por

Donald Schön sobre el Taller de

Proyecto poniendo en cuestión su

afirmación sobre la posibilidad de

aprender pero no de enseñar diseño.

Para fundamentar nuestra posición no

sólo caracterizaremos la mirada de

Schön como la de un extraño sino

también recurriremos a otras voces

autorizadas sobre el tema, voces que

provienen tanto de diseñadores como

de docentes de diseño. A los efectos

de ampliar nuestros argumentos

señalaremos también lo que

permanece invisible a la mirada de

Schön: las teorías de y sobre el

Cuadernos del Centro de Estudios de Diseño y Comunicación Nº 67

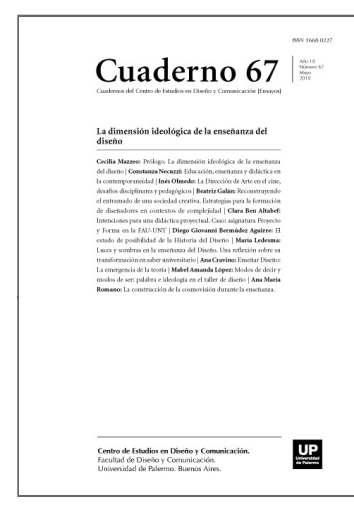

ISSN: 1668-0227

La dimensión

ideológica de la

enseñanza del diseño

Año XVIII, Mayo 2018, Buenos Aires,

Argentina | 260 páginas

descargar PDF

ver índice de la publicación

Ver todos los libros de la publicación

compartir en Facebook

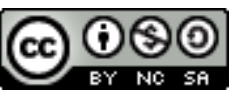

Esta obra está bajo una Licencia Creative

Commons Atribución-NoComercial-

Compartirlgual 4.0 Internacional

Diseño, teorías, que vale la pena

decir, deben ser enseñadas. Y por último describiremos aquello que a Schön le sorprende: el aprendizaje por definición y resolución de problemas, asunto que es presentado por muchos pedagogos como una innovación educativa pero que en el taller de Diseño se hace desde hace muchísimo tiempo...

Palabras clave:

Enseñanza del diseño - tradiciones de enseñanza - didáctica del diseño - taller de diseño - teoría del diseño.

${ }^{*}$ ) Arquitecta, UM. Profesora Superior Universitaria, UM. Magister en gestión de proyectos educativos, CAECE.

Doctora en Arquitectura, FADU-UBA. Docente e Investigadora de las Universidades de Buenos Aires y Palermo.

\section{Aclaraciones preliminares: refutando a Donald Schön}

En su clásico libro sobre la Didáctica Domingo Contreras (1994, p. 14) sostiene con aparente obviedad que "La Didáctica se ocupa de la enseñanza, o más precisamente, de los procesos de enseñanza aprendizaje”, pero avanza con mayores precisiones cuando afirma que la enseñanza: "es una práctica humana que compromete moralmente a quien la realiza" y que a su vez es una actividad social porque sus funciones "están más allá de 
las intenciones y previsiones individuales de los actores directos en la misma, necesitando atender a las estructuras sociales ( ) para comprender su sentido social”. Esta doble dimensión: individual y social es entonces una característica esencial de la enseñanza. No se puede entender una sin la otra, ya que, si bien el aprendizaje se da en el interior de cada sujeto, la enseñanza es en todo social: sus prácticas se constituyen dentro de un sistema de reglas y convenciones que periódicamente son redefinidas. Es por ello que diversos textos sobre Didáctica comiencen con algunas preguntas iniciales que necesitan ser respondidas: “¿Cómo se enseña? ¿Cómo se aprende? ¿Qué se aprende?"

Hace ya unos años Donald Schön (1998, p. 29) concluyó un análisis sobre la formación de profesionales afirmando taxativamente que el Diseño es una competencia que no se ense- ña sino que se aprende mediante el auxilio de un tutor guía. Esta sentencia audaz inquietó a muchos, fundamentalmente a los profesionales de Diseño, dentro de los cuales no se encontraba efectivamente Schön. El era un graduado de Filosofía, ajeno entonces a la práctica proyectual, que visitaba un taller de Diseño para observar su dinámica. Como todo profesional formado en una disciplina, conoce las tradiciones de la misma, entre las que se encuentran los modelos de enseñanza y aprendizaje, que en el caso de la Filosofía, apelan a la clásica clase magistral y al libro de texto. El mismo Schön (1998, p. 41) sostiene que "una práctica profesional es la competencia de una comunidad de prácticos que comparten, en palabras de John Dewey, las tradiciones de una profesión. Comparten convenciones de acción que incluyen medios, lenguajes e instrumentos distintivos". Recordemos, por otra parte, que la Sociología de la Ciencia, se interesa por los aspectos sociales de la actividad científica y aborda, entre otras cuestiones, la manera en que se organiza socialmente una comunidad disciplinar tanto en la producción y difusión del conocimiento como en la selección y entrenamiento de sus miembros. En este sentido es interesante señalar que Steven Shapin y Simon Schaffer, (2006) adherentes al Programa Fuerte, la versión más radicalizada de la Sociología de la Ciencia, afirman que:

Si pretendemos ser extraños a la cultura experimental, podemos buscar apropiarnos de una gran ventaja que ésta posee frente a los miembros de esa cultura, explicando creencias y prácticas de la cultura específica de la que se trata: el extraño está en una posición adecuada para saber que hay alternativas a esas creencias y prácticas.

Pero advierten explícitamente: “jugar a ser extraños, no ser extraños”. (Shapin - Schaffer, 2006, p. 33) El enfoque de Schön está sesgado por la fascinación, y también por el desconocimiento. Su mirada es -en efectola de un extraño. Hemos caído (¿Quién no?) en la trampa formulada por Schön, quizás simplemente sobre su seductor elogio de la práctica de diseño y del taller de arquitectura (Cravino, 2009). Elogio que sentimos que, como diseñadores, nos debería alcanzar... Es, entonces, la intención de este escrito reflexionar sobre la didáctica del Diseño, aunque muchas veces lo haremos abordándolo desde la didáctica del Diseño Arquitectónico o del Proyecto Arquitectónico, simplemente sobre la base de que éste última podría considerarse un caso de la primera y porque la tradición de enseñanza de esa disciplina posee más antigüedad y está más institucionalizada. Necesitamos volver a Domingo Contreras (1994, p. 18) quien alega que "la Didáctica es parte del entramado de la enseñanza y no una perspectiva externa que analiza y propone prácticas escolares". Como ya hemos afirmado oportunamente (Cravino, 2012) (Cravino, 2015): la enseñanza de una disciplina es parte del conjunto de tradiciones que configura esa disciplina. Y las instituciones de enseñanza "son deudoras de una historia" aunque las "sucesivas generaciones que participan en ellas, las vivan como objetivas" (Contreras, 1994, p. 26), es decir, como dadas y permanentes. 
Recordemos entonces algunos conceptos vertidos por Schön sobre los que coincidimos y detengámonos sobre otros que conviene revisar: Concordamos en los siguientes puntos:

- Un diseñador juega con variables, reconcilia los valores en conflicto y transforma los impedimentos. Se trata de un proceso en el que, aún a sabiendas de que unos productos diseñados pueden ser mejores que otros, no existe una única respuesta válida. (Schön, 1998, p. 49) - Un diseñador es "alguien que transforma situaciones indeterminadas en determinadas ( ) Los diseñadores construyen e imponen una coherencia propia. ( ) El análisis y la crítica juegan un papel relevante dentro de un proceso más amplio". (Schön, 1998, p. 50) - El maestro de un taller se da cuenta de que éstos (los estudiantes) no pueden, al principio, comprender las cosas más básicas. Luego se da cuenta de que no puede explicar estas cosas con la esperanza que comprendan, al menos al principio, porque solamente pueden irse captando por medio de la experimentación del propio proceso de diseño. (Schön, 1998, p. 85) - Los profesionales del diseño ( ) tratan con la incertidumbre, la singularidad y el conflicto. Las situaciones no rutinarias de la práctica resultan, al menos en parte, indeterminadas y se les debe proporcionar algún tipo de coherencia. ( ). De vez en cuando, sus esfuerzos para poner orden en una determinada situación provocan resultados imprevistos ( ). Este conjunto de cuestiones -la reformulación del problema, el experimento riguroso, el descubrimiento de consecuencias e implicaciones, la charla retrospectiva y las respuestas a este tipo de charla- es el que configura una conversación reflexiva con los materiales de una situación: el arte de la práctica profesional que caracteriza el diseño". (Schön, 1998, p. 146)

De igual modo agrega Schön (1998):

La paradoja de aprender una competencia nueva es la siguiente: que un estudiante no puede, al principio, comprender lo que necesita aprender, sólo puede aprenderlo formándose a sí mismo y sólo puede formarse a sí mismo comenzando por hacer lo que aún no comprende. (p. 93)

Pero esta paradoja de no comprender lo que necesita conocer se da en todas las disciplinas... Popper (2005, p. 195) afirma "Sólo hay una manera de aprender a comprender un problema que no hayamos comprendido todavía: tratar de resolverlo y fracasar. ( ) Y comprender un problema no es otra cosa que aprehender en qué consiste esa dificultad particular".

Sin embargo, no estamos de acuerdo en que este proceso es "algo factible de ser aprendido y tutorizado, pero no enseñado" (1998, p. 146), y que el estudiante aprende "formándose a sí mismo", como si pudiera prescindir del docente, aquel, que, al fin y al cabo, lo impulsa a hacer "lo que aún no comprende", cuestión que no podría abordar por sí sólo. ¿Será entonces que el docente sí entiende qué debe hacer y por qué debe hacer un determinado ejercicio el alumno...? ¿No será que es profesor quien selecciona las prácticas "formativas" a las que se enfrenta el estudiante? Recordemos que las estrategias de enseñanza están constituidas por todo el conjunto de decisiones que toma el docente, orientando su enseñanza conscientemente, con el propósito de promover el aprendizaje de sus discípulos. Si bien es cierto lo que Schön sostiene respecto a que:

- Un proceso de diseño competente es una forma de conocimiento en la acción. ( ) Esto ayuda a explicar por qué los estudiantes deben practicar si quieren aprender a diseñar -y sugiere, además, que su práctica debe implicar la reflexión en la acción- pero no explica por qué no pueden aprender a diseñar en el orden propio de un currículum profesional normativo: primero teorías en el aula, después un prácticum que las aplica. - Diseñar es una habilidad integral.( ) Por lo tanto, uno no puede aprender de una forma atomizada, aprendiendo primero a 
desarrollar unidades más pequeñas y luego a ensamblar esas unidades en un proceso de diseño global1; pues las piezas tienden a interactuar entre sí y a derivar sus significados y consecuencias del proceso global del que están formando parte. - Un proceso competente de diseño depende de la habilidad del diseñador para reconocer y apreciar cualidades del diseño deseables y no deseables. - Lo que es cierto de la descripción y el reconocimiento de las cualidades del diseño lo es, también, en términos generales, para la descripción y el reconocimiento de un diseño competente. - La descripción del propio conocimiento en la acción que uno posee, es en sí misma, una competencia, y los diseñadores pueden poseerla en mayor o menor medida. Los diseñadores pueden aprender a mejorar las descripciones del diseño -a hacerlas más complejas, precisas y útiles para la acción- por medio de una reflexión continuada sobre sus propias ejecuciones competentes. Diseñar es una actividad creadora. La conversación reflexiva que un diseñador establece con los materiales de una determinada situación puede generar nuevos descubrimientos, significados e invenciones. (Schön, 1998, pp. 146-149)

Aceptar que el Diseño es "una forma de conocimiento en acción", "una habilidad integral" y una "actividad creadora", no implica en modo alguno que no pueda enseñarse.

Pero ¿por qué esta confusión?

La singularidad del Diseño y su enorme complejidad puede dar lugar a simplificaciones y reduccionismos... Es del todo cierto que el docente de la asignatura Diseño no utiliza con frecuencia la clase magistral y, a veces, no recurre a material bibliográfico de apoyo, sin embargo esto no significa que la disciplina Diseño tomada en todas sus acepciones posibles (Diseño de indumentaria, de paisaje, de imagen y sonido, de interiores, textil, industrial e incluso arquitectónico) no tenga un marco teórico, un vocabulario específico y unas precisas herramientas metodológicas que necesariamente deben ser enseñadas.

Asimismo, Schön está confundiendo una materia de un plan de estudio -el taller de Diseño- con la totalidad del campo disciplinar del Diseño. La evidencia de ello surge de la afirmación que sostiene que el proceso de diseño "no explica por qué no pueden aprender a diseñar en el orden propio de un currículum profesional normativo: primero teorías en el aula, después un prácticum que las aplica", aunque él mismo reconoce "el diseño es una habilidad integral". Si el Diseño es una habilidad "integral” ¿que sería aquello que se debe integrar? ¿Acaso, los planes de estudio de Arquitectura y Diseño no contienen otras asignaturas que expresan tácitamente un marco teórico y responden al modelo clásico de "racionalidad técnica"...? ¿Y estas asignaturas no incluyen, acaso, contenidos conceptuales y procedimentales que deben ser "integrados" en el taller de Diseño? Si no fuera así: ¿para que existirían estas asignaturas?

Tengamos presente también lo que decía en 1968 Christopher Jones (1977) sobre el acto de imaginación:

La imagen creativa del diseño, la del diseñador como un mago, es una imagen poética de lo que hay tras cada acción de un ser, humano o animal, provisto de sistema nervioso. Es pues, racional creer que las acciones aprendidas estén controladas inconscientemente, e irracional pretender que el diseño sea susceptible de explicación racional. (p. 4)

Es por ello que el proceso del diseño no puede ser explicado ni comprendido en términos meramente lógicos y racionales, (lo que Schön llama "modelo de racionalidad técnica") y esto ocurre no sólo en el camino del 
aprendizaje sino también en la propia práctica profesional, práctica que le es ajena a Schön.

Alberto Pérez Gómez va un poco más allá y pareciera contestarle a Schön cuando sostiene enfáticamente: "Sí se puede enseñar a proyectar, pero debe tenerse en consideración que se puede enseñar siempre y cuando no entendamos a la escuela como una simulación de la práctica" (Aguilera González - Ayllón Ortiz, 2014, p. 17) Es de gran importancia esta sentencia, no sólo porque la primera parte de la aseveración apoya nuestros argumentos, sino porque la segunda plantea una crítica severa a cierta tendencia de la enseñanza actual que confunde los objetivos de una práctica educativa orientados al aprendizaje, con una supuesta función social del producto de esa práctica. Aclaremos un poco: Cuando un estudiante diseña un hospital, una camilla, un afiche, no está resolviendo un hospital, una camilla ni un afiche: Está aprendiendo a hacerlo bajo la tutela de un profesor. Es falso y también es perverso decir que los alumnos van a resolver un problema real -arquitectónico, espacial, objetual o comunicacional-. Si fuese así se estaría igualando el aprendiz con el profesional, el lego con el experto. Los ejercicios que se desarrollan en el taller de Diseño están fundamentados por objetivos pedagógicos, incluyendo entre ellos, obviamente, la educación en valores. Muchas veces estos ejercicios, por razones didácticas, reducen las variables en juego, simplemente para facilitar el aprendizaje. Otras veces, las complejizan, para exponer al estudiante ante diversas situaciones. Es por ello que ofrecer los productos de los alumnos a la sociedad o al mercado profesional es dañino para los estudiantes, para los profesionales y para toda la comunidad. La única justificación de ello es el trabajo no remunerado de los aprendices y un cierto marketing deshonesto de algunas pocas instituciones educativas confundidas respecto a su verdadera función...

Estas cuestiones nos llevan a otras preguntas: ¿cómo se enseña? ¿En qué consiste este "diálogo reflexivo" que sorprende a Schön? ¿El docente es un tutor que acompaña, a la manera del método socrático a que el alumno construya su conocimiento? ¿El conocimiento está entonces oculto en el interior de cada estudiante, y cada estudiante puede reconstruirlo desde cero? ¿O acaso el docente, en este diálogo aparentemente desprovisto de ideología y de carga teórica, no "baja línea", no expresa una serie de creencias y principios? ¿Para facilitar el aprendizaje del alumno y la construcción de conocimiento, este docente no realiza un conjunto de "transposiciones didácticas", definidas por Yves Chevallard (1991, p. 45) como "el conjunto de las transformaciones que sufre un saber con el fin de ser enseñado", lo cual no sólo consiste en hacer un recorte de lo que se puede enseñar sino una transformación intencionada que efectúa un docente para la enseñanza.

Tomemos un ejemplo sencillo: Comparemos la serie de referentes que posee el alumno al comienzo de su formación universitaria y la que presenta unos pocos meses más tarde. Preguntémosle a ese alumno que justifique su selección y encontraremos un incipiente marco conceptual que actúa a la manera de paradigma: Que ve y que dejó de ver. Dice Barry Barnes (1986) que:

Kuhn describe justamente la formación como proceso de socialización. Transmite no tan sólo las pautas singulares e inherentes a la naturaleza física; también las pautas de convenciones que constituyen una subcultura. La credibilidad se origina ( ) principalmente en la autoridad. (p. 54)

Este proceso de socialización se da en el taller de Diseño, en donde el docente, que es más que un simple tutor guía, se configura como una autoridad. Para corroborar esto no hace falta más que recordar la función del maitre d'atelier en las Escuelas de Bellas Artes. Esta relación docente-alumno, no es la clásica del proceso de enseñanza y aprendizaje habitualmente tipificada en el aula escolar. En el taller, el alumno se "entrega" al docente, para que éste lo forme. Este acto de entrega -del estudiante al docente- tiene éxito en cuanto se funda 
en la búsqueda de beneplácito y consentimiento, cuando se sustenta en la autoconciencia que el alumno tiene acerca de lo que él mismo debe aprender y de lo que el profesor tiene para enseñar, y no sólo se basa en el simple deseo de aprobación de una asignatura o en la mera transferencia de información. Es por ello que es fundamental la presencia de docentes que no sean simples "enseñantes", sino que sean tomados como "modelos" profesionales: objetos de admiración para el conjunto de estudiantes. El propio Schön (1998, p. 117) lo reconoce, aunque no profundiza, cuando define la imitación reflexiva como "una disposición a hacer como lo está haciendo el maestro..."

El método pedagógico del Atelier de la École de Beaux Arts no difiere mucho de cualquier taller actual de Diseño y desciende de atelier renacentista de pintores y escultores. Corona Martínez (1990) lo define así:

El taller es un mundo en el que la arquitectura se define a su modo, y por eso mismo un ambiente que lo satisface a condición de que los herejes sean expulsados periódicamente; los mejores docentes son a larga los que se entienden con menos palabras con el titular, en un «juego de equipo», no necesariamente los más didácticos, puesto que los alumnos juegan un rol secundario en el Taller: reflejan bajo mil formas diferentes la versión de la Arquitectura que sus profesores impulsan, son espejos en los que se ajusta la imagen de sí que tienen los docentes, manos que hacen sus proyectos.

De manera que, para Corona Martínez son los docentes, y fundamentalmente el profesor titular de cátedra, el que le pone la impronta a la enseñanza del taller.

Reflexionemos sobre una expresión que aparece repetida en Corona Martínez y Schön: la noción de "espejo". Para Corona Martínez, como el auxiliar docente intenta imitar o reflejar al profesor titular, el alumno intenta parecerse a ambos. De ahí surge la noción de "Manierismo": "hacer a la manera de". Schön sostiene en cambio que:

En la sala de espejos, estudiante y tutor cambian continuamente de perspectiva. En un determinado momento ven su interacción como una reconstrucción de algún aspecto de la práctica del estudiante; en otro, como un diálogo sobre esa práctica; y en otro más un modelado del rediseño.

Nos queda una duda: ¿En qué momento el alumno asume la perspectiva del docente? Por otra parte, en muchas disciplinas y no sólo en las del Diseño, hay un momento de metacognición donde el profesor intenta hacer conciente en el estudiante lo que ha aprendido... Asimismo ¿qué es eso del "modelado del rediseño"? Aprender y enseñar a diseñar son procesos distintos y dinámicos donde todo está sujeto a revisión y a reflexión.

Entonces ¿Por qué Schön no ve esto? La respuesta es obvia: porque fue formado en otro paradigma.

Dice Schön (1998, p. 129): "La comunicación sobre diseño está sujeta a los impedimentos de la ambigüedad, la falta de concreción y la falta de claridad". ¿Es realmente así? ¿Cómo es posible establecer un "diálogo reflexivo" si el supuesto tutor utiliza un lenguaje ambiguo y poco preciso? ¿No será que Schön no entiende el lenguaje del taller porque no comparte la tradición de una formación? ¿A qué llama "falta de concreción", si, a diferencia de otras disciplinas, el proyecto tiene una concreción y materialidad?

Por lo demás, ¿Qué es lo que le pasa desapercibido a Schön? ¿Y qué es eso que lo maravilla? Para Schön, el diálogo reflexivo que se establece entre el alumno y el docente es una manifestación del rol secundario del tutor 
que "sólo" guía al estudiante a construir su aprendizaje, pero Schön no ve la importancia de la mano del docente que traza sobre el trabajo del alumno correcciones que para ambos son significativas, aunque no lo son para el visitante "extranjero". Ese gesto, aparentemente mínimo, está cargado de valor y puede decir muchas cosas. Una simple raya trazada sobre el dibujo de un estudiante puede ser un estímulo, pero también puede ser una reprobación y una censura. No sólo se establece entre el alumno y el profesor una conversación verbal, hay otro tipo de comunicación, y quizás es la más importante. El profesor a veces no escucha al alumno: "lee" las intenciones en su trabajo. Podemos ver el problema que se presenta en Schön, que Kuhn no dudaría de calificar de "inconmensurabilidad entre paradigmas", en un debate del Consejo Directivo de 1927, cuando la carrera de arquitectura estaba incluida junto a la ingeniería en la Facultad de Ciencias Exactas, Físicas y Naturales de la Universidad de Buenos Aires:

El entonces consejero Ing. Castello manifiesta su disconformidad con las evaluaciones de los trabajos del taller y cuestiona que:

Los exámenes generales de Arquitectura se daban sin la presencia del alumno: es realmente inadmisible que suceda eso en ningún examen de ninguna materia y mucho menos para Arquitectura en que únicamente el alumno puede certificar si ha hecho él el trabajo o no.

A lo que responde el consejero por la Escuela de Arquitectura, Arq. Ezequiel Real de Azúa señalando que:

Los trabajos de Arquitectura -hablo con experiencia personal-son de los pocos que se pueden tal vez analizar sin que se pregunte nada al autor, tanto que en un concurso se determina el mejor trabajo sin la presencia del autor. ( ) Hay alumnos que hablan por los codos sobre un trabajo que no han hecho, y otros que no son capaces de hablar sobre el mejor trabajo que han hecho"2 .

Lo que resulta obvio para un arquitecto, no lo es para un ingeniero.Vayamos a la tercera pregunta donde se encuentra el foco del problema: ¿Qué es lo que se aprende? Recordemos a Schön: Diseño "es una habilidad integral" y "una actividad creadora". Es cierto, pero no. El Diseño no es sólo una habilidad, no es sólo una actividad, no es una mera destreza, no es únicamente un saber hacer, una reflexión para la práctica. Es algo más. Mucho más. Tenemos que decirlo: Hay un marco teórico construido. Y hay un sistema de valores que debe ser enseñado. Y hay un conocimiento disciplinar. La reflexión en la acción, podríamos declararle a Schön, produce conocimiento que va más allá de la propia acción. (Mazzeo - Romano, 2007, p. 32) (Fernández, 2013) (Sarquis, 2003) (Ledesma, 2005)

\section{La necesidad y urgencia de las Teorías de y sobre el Diseño}

Roberto Fernández (2013, p. 31) señala que en la actualidad el campo de la arquitectura presenta "un extremo desprecio por la condición reflexiva propia de la producción de teoría", agregando luego que:

La debilidad de la teoría - dentro del espectro cognitivo de la arquitectura- no solo afecta las modalidades de enseñanza (hoy más orientadas a cubrir las expectativas contingentes de tal frivolidad: aquello que Derrida definía como interés por los significantes y desinterés por los significados. Podría rastrearse cómo esta dualidad se distribuye dentro del pensum actual de la enseñanza) sino que compromete la actividad y función misma de la investigación cuyos emergentes deben contribuir a fortalecer la teoría. 
Lo mismo ocurre en el campo del Diseño Gráfico, ya que como Leonor Arfuch (2003, p. 10) sostiene "hay un aspecto que aparece reiteradamente en unas y otras posiciones: la falta de teoría, de una reflexión propia o pertinentemente «apropiada» de ciertas disciplinas afines, la carencia de un aparato crítico, la escasa articulación con otros saberes". Esta ausencia -o rechazo- de la teoría se despliega por todas las disciplinas proyectuales, justificada, a veces, por la urgencia y el deseo de hacer, y, también, por la devaluación de la cultura escrita que sacraliza a la práctica y cuestiona a la teoría, presuponiendo tácitamente que la segunda es aburrida y difícil, y la primera divertida...3 También esta justificación de falta de teoría se alimenta en la exaltación de la pura intuición y el mito de la inspiración creadora, cuyo ejemplo más visible es la idea de partido. Sin embargo, vale reafirmar que, revalorizar la teoría no implica, en modo alguno, dejar de lado el enfoque constructivista del aprendizaje iniciado por Dewey y la importancia del "aprender haciendo" Recordemos lo que Sergio Martínez Muñoz (1999, p. 506) sostiene "Las teorías no existen en ningún paraíso platónico o estructuralista, más bien deben verse como construcciones intelectuales apoyadas en un contexto de prácticas que se estructuran en tradiciones intelectuales que tienen una historia". Este "contexto de prácticas" y estas "tradiciones intelectuales" encuentran su lógico fundamento en la enseñanza. Vale señalar que cuando las teorías en cuestión no aparecen explicitadas, están presentes a la manera de un mensaje oculto que fundamenta una determinada posición ideológica. Recordemos que la ideología sería para Verón (1969) el metamensaje oculto que posee cada mensaje, la parte no manifiesta, ni a veces consciente, pero plagada de valores, creencias y conceptos, que de alguna manera guía nuestro accionar. Es claro entonces que todo mensaje tiene metamensaje, que todo discurso -incluso el más pueril- tiene su carga ideológica y normativa: la enseñanza no es la excepción. Tengamos en cuenta también que Gregorio Kimovsky (1975, p. 13) considera que uno de los sentidos que puede darse al concepto ideología es el de marco teórico, que es tomado de manera tácita como "conjunto de conceptos y presuposiciones al que un científico tiene recurrir para desarrollar una teoría..." Es necesario comprender que, a partir de Thomas Kuhn e Imre Lákatos, las teorías dejan de ser un mero conjunto de hipótesis para constituir una estructura, lo cual se fundamenta según Alan Chalmers (1988, p. 114) por dos motivos: "el hecho de que el estudio histórico muestra que las teorías científicas poseen esa característica y el hecho de que los conceptos solamente adquieren un significado preciso mediante una teoría coherentemente estructurada". En el campo específico de las reflexiones sobre una Teoría del Proyecto en arquitectura encontramos a Jorge Sarquis (2003a) quien a su vez cita a Immanuel Kant que afirmaba respecto a la presunta dicotomía teoría-práctica que:

Se denomina teoría incluso a un conjunto de reglas prácticas, siempre que tales reglas sean pensadas como principios, con cierta universalidad, y por lo tanto siempre que hayan sido abstraídas de la multitud de condiciones que concurren necesariamente en su aplicación.

Estas afirmaciones pueden extenderse a la teoría de cualquiera de los diseños.

Por otra parte, Deleuze respondiendo a Foucault quien había afirmado que "la teoría no expresa, no traduce, no aplica una práctica; es una práctica" sostiene, entonces, vaciando de contenido a la teoría, que la misma es "como una caja de herramientas..." (Foucault, 1999, p. 107)

Según Sarquis (2003b, p. 32) Quatremère de Quincy encuentra tres niveles en el sentido de "teoría":

1. "La teoría práctica «de los hechos y de los ejemplos»", es lo que Quintanilla (1991, 38) denomina "conocimiento operacional o conocimiento práctico" que consiste en "el conjunto de instrucciones ordenadas que 
hay que seguir para obtener un resultado determinado a partir de una situación dada". El punto de partida de este conocimiento es el estudio de casos, la observación, la experimentación y la propia práctica, recurriendo habitualmente al método de ensayo y error. Esto constituye lo que se denomina "saber técnico", es decir aquel tipo de conocimiento que incluye desde habilidades y destrezas aprendidas en la práctica, no verbalizables, hasta modelos o procedimientos a imitar para alcanzar una meta. Esta teoría es la que se emplea para enseñar a dibujar y cuya competencia se adquiere en la práctica. Pero el campo del Diseño no se agota en este "saber dibujar"

2. "La teoría didáctica, aquella de «las reglas y los preceptos»" consiste en lo que Quintanilla (1991, p. 39) llama conocimiento representacional de regularidades y leyes. Por otra parte en este punto se encuentra la base del sistema educativo, es decir la sistematicidad pedagógica y el carácter científico de la enseñanza. Por ejemplo en la enseñanza academicista era de gran valor la subordinación a la regla y el apego a la tradición. De ahí la importancia que adquiere la "autoridad", es decir aquel que define las reglas. Como afirma Liernur (2001, p. 92) el saber de la Academia se sostiene entonces en los principios de "erudición y censura: Aprender las leyes; Obedecer las reglas". Lo opuesto es la incertidumbre y en el pensamiento positivista no había lugar para ella. Una ejemplificación de la aplicación de esta teoría en la actualidad es cuando se introduce al alumno la noción de orden, empleo de tramas y operaciones de simetría, así como las leyes de la Gestalt y las reglas de armonía del color.

3. "La «teoría de los principios de las razones sobre las que se apoyan las reglas, y que es llamada teoría metafísica»", que actualmente configuraría la epistemología de una disciplina, entraría en el campo de las metateorías. Al decir de Bunge (1983):

Una metateoría es una teoría acerca de alguna teoría o clase de teoría. Y una teoría o sistema deductivo es a su vez un conjunto de proposiciones ( ) ordenadas por la relación de deducibilidad. Así pues en una teoría no existe ninguna proposición aislada: toda proposición es o una hipótesis o bien una conclusión. (p. 226)

Para aclarar un poco más estos conceptos recurriremos a Ludwig Wittgenstein (1988) quien reflexiona sobre la adquisición de diferentes tipos de saberes, puesto que considera, por ejemplo, que entender un lenguaje significa dominar una técnica. $Y$ dominar una técnica implica saber hacer cosas. Todo juego de lenguaje se aprende, entonces para Wittgenstein, como hecho práctico. Se aprende a hacer algo, a medida en que se lo va haciendo y se cree en lo que se hace. En este punto Wittgenstein y Schön parecieran coincidir. No obstante, para Wittgenstein sólo actuando se aprende un juego de lenguaje determinado y se conocen sus pautas, pero estas pautas no son ajenas a la práctica del propio juego, puesto que se fundamentan como reglas prácticas por la propia práctica. De modo que, todo conocimiento se acepta a partir de la práctica básica que "autoriza" aprender cómo es el mundo. Y esa práctica es la que define y construye ese mundo. No se aleja de este pensamiento Nelson Goodman (1993). Para Wittgenstein, entonces, la certidumbre es consecuencia de la acción y no de la reflexión o conocimiento formal de ciertas reglas. Las creencias, como manifestación de los fundamentos de un juego del lenguaje, siempre se expresan como una práctica con sentido. La duda aparece después de la creencia, nunca antes. Thomas Kuhn (1982, p. 19), también recurre al ejemplo de la enseñanza de un lenguaje, retomando asimismo lo expresado en su "Posdata" de La Estructura de las Revoluciones científicas, donde destaca el valor de los ejemplos y la práctica: 
A los científicos no se les enseñan definiciones, pero sí formas estandarizadas de resolver problemas seleccionados...() Ese procedimiento me pareció muy semejante al empleado para que los estudiantes de idiomas aprendan a conjugar verbos y a declinar nombres y adjetivos....( ) En inglés esos ejemplos estandarizados reciben el nombre de «paradigmas».

En este punto Wittgenstein y Kuhn4 se volverían a cruzar con Donald Schön (1998, p. 93) quien explica el aprendizaje del diseño:

La paradoja de aprender una competencia nueva es la siguiente: que un estudiante no puede, al principio, comprender lo que necesita aprender, sólo puede aprenderlo formándose a sí mismo y sólo puede formarse a sí mismo comenzando por hacer lo que aún no comprende.

Concluyendo con la afirmación que "un proceso de diseño competente es una forma de conocimiento en la acción”. Barnes (1986) también es de la misma opinión, pero refiriéndose al aprendizaje de la ciencia: Se aprende en base a rutinas estandarizadas, las definiciones verbales bien después....

Vale aquí hacer una acotación respecto a las nociones de Poiesis y Praxis, bien diferenciadas por Sarquis (2003 a y b). La acción poiética deja un producto material y sensible, y tiene en la estética un componente esencial, no así la praxis.

Por otra parte Adolfo Corona Martínez (1990), no lejos de Schön, da cuenta de la producción de conocimiento en la práctica proyectual:

El diseñador inventa el objeto en el acto mismo de representarlo; esto es, dibuja un objeto inexistente, cada vez con mayor precisión. Esta precisión es un aumento en el detalle, dentro del sistema de reglas de la representación misma. Así el diseño es la descripción progresiva de un objeto que no existe al comenzar la descripción.

Muy parecida es la definición de Bonta (1977, 34): "se entiende por proyecto una documentación que describe un objeto inexistente y, eventualmente, describe también las operaciones necesarias para conferirle existencia". Es por ello que el proceso proyectual consiste en la construcción de un conocimiento preciso de aquello que no existe, que aún no existe; pero puede existir, gracias a esa misma práctica proyectual. De este modo el "saber por experiencia", único e intransferible, no verbalizable, pero efectivo, a través de la práctica y la empiria, se va transformando en un "saber hacer", lo cual permite establecer algún tipo de regularidad, secuenciación o instrucción, no fundamentada todavía y aparentemente irreflexiva, pero producto de la práctica. Entre uno y otro hay un salto inductivo informal que permite ir del caso a la norma, lo que Barnes denomina "máquina inductiva" o lo que define Arfuch como "pensar el hacer" que es más que reflexionar la acción.... El "saber qué", conceptual o verbalizable, surge de la validación y fundamentación de las normas o regularidades antes mencionadas, de tal modo que adquieren el estatus científico de "ley" configurando así el primer paso de una teoría consolidada. (Ver Figura 1) Respecto al clásico tópico en el que se afirma que "Tal vez esto sea correcto en teoría, pero no sirve para la práctica" sentencia Immanuel Kant (1993, p. 4):

Por tanto, cuando la teoría sirve de poco para la práctica, esto no se debe achacar a la teoría, sino precisamente al hecho de que no había bastante teoría, de modo que el hombre hubiera debido aprender de la experiencia la 
teoría que le falta. ( ) Así pues nadie puede hacerse pasar por prácticamente versado en una ciencia y a la vez despreciar la teoría, sin reconocerse ignorante en su especialidad, por cuanto cree que con tanteos y experimentos realizados a ciegas puede ir más allá del punto hasta donde la teoría es capaz de conducirle, sin hacer acopio de ciertos principios (que constituyen, propiamente, lo que se denomina teoría) y sin haber considerado globalmente su quehacer (lo cual, cuando se procede metódicamente, se llama sistema).

Es por ello que para Kant todo problema práctico se resuelve con más teoría... Lo mismo diría Charles S. Pierce quien sostiene que la abducción es "el único tipo de argumento que da lugar a una idea nueva" (Eco-Sebeok, 1989, p. 40), afirmando luego que la "abducción arranca de los hechos, sin tener, al inicio ninguna teoría particular a la vista, aunque está motivada por la sensación de que se necesita una teoría para explicar los hechos sorprendentes ( ) La abducción busca una teoría” (Eco-Sebeok, 1989, p. 47). Lo que Pierce y Eco coinciden en llamar abducción sería aquello que Jones (1977, p. 3) denomina "caja negra".

Por otra parte, Giddens (1987, p. 163) afirma que toda ciencia es hermenéutica porque exige una dimensión de interpretación y, subsiguientemente, una teoría de decodificación, agregando luego que las ciencias sociales poseen una doble hermenéutica porque el investigador significa lo ya significado por los actores sociales. Extrapolando estas afirmaciones al campo del diseño podemos también allí encontrar esta doble hermenéutica, puesto que la producción de diseño implica una interpretación y análisis de los datos de la realidad transformando lo indeterminado en determinado5 utilizando un método abductivo según la clásica caracterización de Pierce (Eco-Sebeok, 1989, pp. 37-40) ; pero también podemos señalar que, es desde la teoría donde se analiza y dilucida este producto ya significado que necesita ser interpretado nuevamente. Sin embargo, a diferencia de la ciencia (que engendra solamente saber) y, al igual que la tecnología, el Diseño tiene un doble producto: el conocimiento (que actualmente se lo califica como "proyectual") y el artefacto o producto diseñado. Este doble producto implica una serie de complicaciones epistemológicas y ontológicas, y, fundamentalmente, didácticas... Atravesando la elaboración que Sarquis (2003b, p. 36) hace de Gilles Ferry con las afirmaciones de Quatremère de Quincy también citadas por Sarquis encontramos que:

Entonces si el objetivo es alcanzar conocimiento, el carácter de la teoría se nos presenta como hipotético, conjetural, siendo que, como afirmamos, la evidencia empírica no siempre es suficiente para decidir entre teorías en competencia. Por ende, los criterios para elegir entre teorías distintas, habiendo fracasado la contrastación crucial, no son reglas según Kuhn (1982, pp. 344-364), sino valores: precisión, coherencia, amplitud, simplicidad, fecundidad.... Asimismo, con respecto a la construcción de conocimiento en Diseño debemos recurrir a Sarquis (2004, p. 38) quien define a la Investigación Proyectual como "una manera especial de realizar proyectos con el objetivo de obtener conocimientos disciplinares. Se caracteriza por encauzar el procedimiento proyectual atendiendo aspectos que no atienden los proyectos profesionales o formativos tradicionales..." Más adelante agrega: "Entendemos por Investigación Proyectual a los procedimientos que en base a determinadas teorías, metodologías y técnicas son configuradoras de formas espaciales significativas e innovadoras, con capacidad de enriquecer los conocimientos disciplinares para la producción arquitectónica". No lejos de Sarquis se encuentra Cesar Naselli (1997) quien define a la Arquitectura en cuatro sentidos: como saber; como conjunto de prácticas; como producto de prácticas y como profesión. Siendo que el primero incluye la noción epistemológica y científica de lo qué es la arquitectura, abarcando a conjunto de fundamentos, hipótesis, teorías y reflexiones elaborados históricamente sobre la disciplina arquitectónica. Los mismos cuatro sentidos pudieran ser aplicados a cualquier tipo de Diseño. En síntesis, tomando las palabras de Oriol Bohigas (1972) quien refiriéndose a esta multidimensionalidad del diseño y de la propia arquitectura señala: 
Un proyecto no es simplemente una exhibición caligráfica ni un objeto expresivo que se agote en sí mismo, a pesar de que el dibujo sea siempre un indicador de contenido y de intenciones no sólo como código directo, sino como transmisor de una poética y hasta de unos métodos. Pero tampoco es la simple ilustración de una teoría arquitectónica, a pesar de que esa teoría debe quedar explicitada en los mismos elementos de representación. $Y$ tampoco es una sucesión indiscriminada de soluciones constructivas, a pesar de que todas ellas están en las condiciones de partida del proyecto y constituyen parte de su base conceptual. Es necesario que la expresión y el contenido coincidan precisamente en la definición de un edificio concreto donde encuentren solución las dificultades de la realidad y la coherencia conceptual, donde dibujo, teoría y tecnología produzcan una arquitectura construible.

En el proceso del diseño, el practicante realiza una síntesis de un conjunto complejo -y a veces indeterminadode variables, para ofrecer una respuesta a un problema, que no es la única posible. En esta síntesis mental donde se va del todo a la parte y de la parte al todo, con una mirada analítica y a la vez totalizadora del fenómeno, el diseñador impone al proyecto una consistencia y una idea de unidad que le es propia.

No lejos de ello Helio Piñón (2006, p. 18) afirma que "sea cual sea el panorama de doctrinas que lo caracterizan, la práctica de la arquitectura adquiere un sentido específico que depende del uso que el autor hace de los principios teóricos y operativos..."

3. Las estrategias didácticas del taller de Diseño:

Recordemos los principios pedagógicos del taller que formula Ezequiel Ander Egg (1999) que parecieran ser escritos por Schön, por Mazzeo o por Romano:

a) El Aprender haciendo, lo cual implica la superación de la división tajante entre formación teórica y formación práctica, una alternativa diferente a la enseñanza "libresca", adquiriendo conocimientos teóricos, métodos, técnicas y habilidades en un proceso de trabajo, dejando de lado además "la clase magistral y del protagonismo del docente, por la formación a través de la acción/reflexión..." Este enfoque no implica, como vemos, la ausencia de teoría, sino su revalorización instrumental; b) Metodología participativa, que incluye el trabajo grupal; c) Pedagogía de la pregunta, contrapuesta a la pedagogía de la respuesta, en la que toma significativa importancia la definición del problema; d) Entrenamiento que tiende al trabajo interdisciplinario y al enfoque sistémico, lo que se relaciona con la integración esencial del diseño; e) Relación docente-alumno queda establecida en torno a la realización de una tarea común; f) Carácter globalizante e integrador de la práctica pedagógica. Esta integración como mencionamos implica vincular la teoría con la práctica; g) Trabajo grupal mediante técnicas adecuadas; h) Integración en un solo proceso tres instancias: docencia, investigación y práctica, es decir enseñanza, aprendizaje y producción de conocimiento. Esto último va a permitir la construcción teórica.

Por otra parte el diseño se asocia comúnmente con el pensamiento complejo el que apunta a que es necesario articular e integrar las distintas dimensiones de lo real, reconociendo por un lado los quiebres y compartimientos del saber, en un contexto en el que la realidad exige enfoques multi, inter y transdisciplinares. Para lo cual se hace necesario establecer una estrategia reflexiva que tiene una intención globalizadora o abarcativa de los fenómenos, que a su vez, reconoce la especificidad de las partes. Es por ello que la caracterización de Schön de 
reflexión e integración en el Diseño es necesaria, aunque no suficiente.. En este sentido vale rescatar los tres principios que establece Morin (2003):

1. El principio dialógico, que encarna dos lógicas contrapuestas pero mutuamente necesarias.

2. El principio recursivo, que rompe con la idea lineal de causa-efecto. El efecto se vuelve causa, la causa se vuelve efecto.

3. El principio hologramático que busca superar el "holismo" -que no ve más que el todo- y el reduccionismo que no ve más que partes-. Aquí es donde se fundamenta el pensamiento estratégico de diseño: análisis y síntesis.

Desde un punto de vista pedagógico se sabe que el aprendizaje complejo implica la integración de conocimientos, la coordinación de ciertas habilidades que son cualitativamente diferentes, además de la transferencia de lo aprendido en un entorno educativo al ámbito de la vida y el trabajo diarios. El interés actual en el aprendizaje complejo se manifiesta en algunos enfoques, fundamentalmente constructivistas, como el estudio de casos y los casos problema. Se habla entonces de resolución colaborativa de problemas (Nelson, 2000), constructivismo y ambientes constructivistas de aprendizaje (Jonassen, 2000), aprender haciendo (Schank-Berman, Macpherson, 2000). Aunque todas estas propuestas tienen elementos distintos manifiestan en común el hecho que desarrollan "tareas auténticas de aprendizaje" basadas en fenómenos o episodios de la vida diaria como impulso para la enseñanza. La idea principal que sustentan estos enfoques es que la realización de las mencionadas tareas ayudan a los aprendices a integrar conocimientos, habilidades y actitudes, los estimulan para que aprendan a coordinar habilidades constitutivas, y facilitan la transferencia de lo aprendido a nuevas situaciones problemáticas. (Cravino, 2014) Sostienen entonces Tishman, Perkins y Jay $(1997,2002)$ que "la transferencia es un fenómeno de pensamiento y aprendizaje humanos. Tiene lugar cuando la gente adquiere conocimientos, estrategias, predisposiciones $u$ otras cosas que se pueden aprender en un contexto y aplicar luego a otro". Lo contrario es el pensamiento pobre o frágil. De ahí la importancia de promover el aprendizaje reflexivo y genuino. Por otra parte, se sostiene que la forma de trabajo en pequeños grupos favorece la construcción de un entorno estimulante donde aprender y compartir, lo cual genera un impacto positivo por el sentido de pertenencia, lo que abre posibilidades a numerosas dimensiones en las que cada estudiante es invitado a aportar, y donde la participación de cada uno se hace necesaria para la concreción de los objetivos planteados. De modo que, después de indagar en estos "nuevos" modelos de aprendizaje, los que actúan en carreras proyectuales se encuentran como aquel personaje de Moliere que "hablaba en prosa sin saberlo", ya que se afirma que "El aprendizaje mediante ejemplos entronca con contextos reales; los alumnos tienen que arreglárselas ante situaciones complejas y pensar como profesionales" (Jonassen, 2000, p. 228), es decir se plantea como una "innovación" pedagógica, aquello que los profesores de arquitectura y diseño, hacen desde siempre... Este proceso innovativo es lo que sorprendía fundamentalmente a Schön. Edith Litwin (1997, pp. 97-98) caracteriza a las configuraciones didácticas como las maneras particulares que despliega el docente para favorecer los procesos de construcción del conocimiento. Esto significa que existe una

Construcción elaborada en la que se pueden reconocer los modos como el docente aborda múltiples temas de su campo disciplinario y que se expresa en el tratamiento de los contenidos, su particular recorte, los supuestos que maneja respecto del aprendizaje, la utilización de prácticas metacognitivas, los vínculos que establece en la clase con las prácticas profesionales involucradas en el campo de la disciplina de que se trata, el estilo de 
negociación de significados que genera, las relaciones entre la práctica y la teoría que incluyen lo metódico y la particular relación entre el saber y el ignorar.

Esto pone en evidencia que existe por parte del docente una clara intención de enseñar, de favorecer la comprensión de los alumnos y de generar procesos de construcción de conocimiento, con lo cual se distinguen claramente de aquellas configuraciones no didácticas, que implican sólo la presentación de información o la exposición de ideas o temas, sin tomar en cuenta los procesos del aprendizaje del alumno. Por otra parte, como menciona Litwin, la teoría siempre está presente y las prácticas metacognitivas parecieran no ser un atributo particular de la enseñanza del Diseño.

\section{El estudio de casos y el caso problema}

La selección de temas para proyectar, y la consideración de los mismos como caso-problema dependerá del momento histórico de la enseñanza de una disciplina

El análisis de casos no sólo es una técnica de investigación, sino también una estrategia educativa. La enseñanza del diseño desde siempre se ha focalizado sobre un caso-problema, cuyo análisis y resolución constituyen el eje central de la experiencia, sabiendo, asimismo que existe más de una opción viable (Wasserman, 1999) (Escribano-Del Valle, 2008). Generalmente se define al estudio de casos como "una experiencia pedagógica de tipo práctico organizada para investigar y resolver problemas vinculados al mundo real, la cual fomenta el aprendizaje activo y la integración del aprendizaje ( ) desde una mirada multidisciplinar". (Díaz Barriga, 2005, p. 62)

En este tipo de aprendizaje, el alumno debe adquirir habilidades de:

- Adquisición y manejo de información: conseguir, filtrar, organizar y analizar la información proveniente de distintas fuentes. - Comprensión de sistemas complejos: capacidad de ver la interrelación de las cosas y el efecto que producen las partes en el todo y el todo en las partes, en relación con sistemas naturales, sociales, organizativos, tecnológicos, etcétera. - Experimentación: disposición inquisitiva que conduce a plantear hipótesis, a someterlas a prueba y a valorar los datos resultantes. - Trabajo cooperativo: flexibilidad, apertura e interdependencia positiva orientadas a la construcción conjunta del conocimiento. (Díaz Barriga, 2005, pp. 64$65)$

Como vemos muchas de las características de esta estrategia educativa coinciden con la aceptación del pensamiento complejo y el proceso de aprendizaje en el taller.

En la definición del proceso de diseño como un aprendizaje bajo la resolución de problemas, encontramos una síntesis de lo ya conocido: respuestas múltiples, sistemas complejos, experimentación, formulación de hipótesis, camino de ida y vuelta, trabajo colaborativo. Estas experiencias de aprendizaje que se definen como "innovadoras" tienen más de cien años de antigüedad en el campo de la arquitectura y el diseño. Por otra parte, otra aparente "novedad" de esta metodología reside en la articulación entre teoría y práctica, y la postulación que todo proyecto es por "naturaleza" teórico-práctico, cuestión que ya hemos mencionado. La diferencia entre "tema" y "problema" consiste en el carácter descriptivo del primero y el resolutivo del segundo. Un profesor presenta un tema, vivienda por ejemplo, que se transforma en problema para el alumno al requerir de las 
herramientas cognitivas de análisis y síntesis para responder a un número complejo de variables en un caso especial de vivienda. David Jonassen (2000, p. 229) sostiene desde un enfoque explícitamente constructivista que: "Dado que la clave de un aprendizaje significativo implica considerar el problema o el objetivo de aprendizaje como algo propio, es necesario proporcionar problemas interesantes, pertinentes y atractivos de resolver". Destacando asimismo que "El problema no debería estar excesivamente constreñido; por el contrario, debe estar definido o estructurado de forma insuficiente, de manera que algunos aspectos del problema resulten inesperados y puedan ser definidos por los alumnos". Es por ello que para Jonassen un problema debe estar formulado de manera incompleta, puesto que el mismo tiene objetivos y formulaciones que no están completamente definidos; posee múltiples soluciones, varias líneas de soluciones o incluso ninguna solución; requiere múltiples criterios para evaluar las soluciones propuestas; presenta incertidumbre a la hora de aclarar cuáles son los conceptos, las reglas y los principios necesarios para una solución dada; no ofrece reglas o principios generales para describir o predecir de manera concluyente el resultado; requiere, asimismo que los estudiantes establezcan juicios sobre el problema y defiendan su caracterización expresando sus opiniones o sus creencias personales. No hay duda que en este aspecto Schon y Jonassen coincidirían. Lo que destaca entonces Schön de la singularidad del Diseño es que el aprendiz se inicia a partir de la simulación de una práctica profesional que se basa en la resolución de problemas, modelo de enseñanza y aprendizaje que es necesario promover. Pérez Gómez enfatizaría la idea de que el estudiante "juega" como profesional pero no debe creerse esta simulación. Sus productos, no son, en modo alguno, productos de un experto. La cuestión que destaca Schön, es que a diferencia del aprendizaje cuyo modelo responde a la racionalidad técnica donde la resolución de un problema es una respuesta instrumental a una situación ya definida, en el campo del Diseño los propios fines y problemas son confusos y complejos, de tal manera que la respuesta alcanzada presupone un nivel de conflictividad que en modo alguno se eliminaría mediante el empleo de herramientas de la racionalidad técnica, ya que esa solución depende de cómo el problema sea definido. Enseñar a diseñar no consistiría entonces en instruir al estudiante para que sea un hábil "solucionador" de problemas, sino en enseñar a ver el conflicto y la complejidad intrínseca de una situación que necesita ser resuelta y cuya solución excede los criterios prácticos de eficacia/eficiencia.

Notas

1. Queda fuera de este trabajo la reflexión sobre el modelo de enseñanza de la Escuela de Bellas Artes de París que sería replicado por todo el mundo y perduraría por casi un siglo: el método de Composición Arquitectónica, donde se iniciaba al alumno en el conocimiento primero de los Elementos de Arquitectura, luego de los Elementos de Composición para concluir luego en la resolución final: La Composición Arquitectónica. Este modelo refutaría también los argumentos de Schön. Ver (Cravino, 2015) 2. Archivos de la Universidad de Buenos Aires, 1927, Tomo III, Pág. 37-38. 3. No es casual entonces que una Universidad publicite su modelo de enseñanza expresado por el slogan "aprender haciendo"... 4. No es casual entonces que el primer libro de Donald Schön The Displacement of Concepts, publicado en 1963, estuviera claramente influenciado por la Historia de las ideas expresada por Thomas Kuhn... 5. Ver Resolución 498 del 2006 de la CONEAU para la acreditación de las carreras de Arquitectura en Argentina. 
Aguilera González, A. y Ayllón Ortiz, J. A. (2014). Alberto Pérez-Gómez. De la educación en arquitectura. Ciudad de México: Universidad Iberoamericana. Ander-Egg, E. (1999). El taller: una alternativa de renovación pedagógica. Buenos Aires: Magisterio del Río de la Plata. Arfuch, L.; Chaves, N. y Ledesma, M. (2003). Diseño y comunicación. Teorías y enfoques críticos. Buenos Aires: Paidós. Aymonino, C. (1981). El significado de las ciudades. Madrid: Ed. Blume. Barnes, B. (1986). T. S. Kuhn y las ciencias sociales. México: F.C.E. Bohigas, O. (1978). Proceso y erótica del diseño. Barcelona: Editorial La Gaya Ciencia. Bonta, J. P. (1979). "Nota sobre los temas discutidos en Portsmouth" en Jones, J. Ch. y otros (1979). El simposio de Portsmouth: problemas de metodología del diseño arquitectónico. Buenos Aires: EUDEBA. Bunge, M. (1983). "Metateoria” en Bunge, M. y Bar-Hilleil, Y. (1983). El pensamiento científico. Conceptos, avances, métodos. Madrid: Tecnos. Camilloni, A W. y otros (1996). Corrientes didácticas contemporáneas. Buenos Aires: Paidós. Camilloni, A. W. y otros (2007). El saber didáctico. Buenos Aires: Paidós. Chalmers, A. (1988). ¿Qué es esa cosa llamada ciencia? Buenos Aires: Siglo Veintiuno. Chevallard, Y. (1991). La transposición didáctica. Del saber sabio al saber enseñado. Buenos Aires: Aique. Corona Martinez, A. (1990). Ensayo sobre el Proyecto. Buenos Aires: Editorial CP67. Cravino, A. (2009). "Releyendo a Donald Schön” en Reflexión Académica en Diseño y Comunicación 2009. Año X, Vol. 11, Febrero 2009, Buenos Aires, Argentina, Pág. 65-69.

Cravino A. (2012). Enseñanza de Arquitectura: Una aproximación histórica. 1901-1955: La inercia del modelo Beaux Arts. Buenos Aires: SCA/Nobuko. Cravino, A. (2014). "Disciplina, integración y transferencia en la construcción del saber proyectual" en rda - revista de la facultad de arquitectura de la ucsf, Universidad Católica de Santa Fe, año 2, № 2, septiembre de 2014. Cravino, A. (2015). Enseñanza de la arquitectura: La rebelión impasible de las disciplinas. Universidad de Buenos Aires, 1897-1956. Tesis doctoral, FADU-UBA, Buenos Aires, inédita. Deleuze, G. y Foucault, M. (1972). "Los intelectuales y el poder” en Foucault, M. (1999) Estrategias de poder. Obras esenciales. Volumen II. Barcelona: Paidós. Díaz Barriga, F. (2005). Capítulo 3 "El aprendizaje basado en problemas y el método de casos" en Enseñanza situada: Vínculo entre la escuela y la vida. México: McGraw Hill. Doberti, R. (2008). “Tipologías semánticas” en Doberti, R. (2008). Espacialidades. Buenos Aires: Ediciones Infinito. Sebeok, T. y Sebeok, J. (1989). "Ya conoce usted mi método». Una confrontación entre Charles S. Pierce y Sherlock Holmes" en Eco, U. y Sebeok, T. (1989). El signo de los tres. Barcelona: Lumen. Escribano, A. y Del Valle, Á. (2008). El aprendizaje basado en problemas. Una propuesta metodológica para la Educación Superior. Madrid: Narcea. Fernández, R, (2013), Inteligencia proyectual Un manual de investigación en arquitectura, Buenos Aires: Editorial Teseo. Giddens, A. (1987). Las nuevas reglas del método sociológico. Buenos Aires: Anirrortu. Goodman, N. (1993) Maneras de hacer mundo. Madrid: Visir. Jonassen, D. (2000), Capítulo 10 “El Diseño de Entornos Constructivistas de Aprendizaje”, en Reigeluth, Ch. (2000). Diseño de la Instrucción: Teorías y modelos. Un nuevo paradigma de la teoría de la instrucción. Madrid: Aula XXI Santillana. Jones, J. Ch. (1979). "El estado actual de los métodos de diseño" en Jones, J. Ch. y otros (1979). El simposio de Portsmouth: problemas de metodología del diseño arquitectónico. Buenos Aires: EUDEBA. Kant, I. (1993). Teoría y Práctica. Madrid: Tecnos. Klimovsky, G. y otros (1975). Ciencia e Ideología. Buenos Aires: Ediciones Ciencia nueva. Kuhn, T. (1982). La tensión esencial. Estudios selectos sobre la tradición y el cambio en el ámbito de la ciencia. México: Fondo de Cultura Económica. Ledesma, M. del V. (2005). Legitimidades y olvidos. Una contribución a la epistemología del diseño. Tesis doctoral. Buenos Aires: FADU-UBA. Liernur, J. F. (2001). Arquitectura en la Argentina. Buenos Aires: Fondo Nacional de las Artes. Linazasoro, J. I. (1981). El proyecto clásico en Arquitectura. Barcelona: Editorial Gustavo Gili. Litwin, E. (1997). Las configuraciones didácticas. Buenos Aires: Paidós. Litwin, E. (2008). El oficio de enseñar. Condiciones y contextos. Buenos Aires: Paidós. Martínez Muñoz, S. (1999). "Otto Neurath y la filosofía de la ciencia del siglo XX" en Perspectivas 
Teóricas Contemporáneas de las Ciencias Sociales. México: UNAM. Mazzeo, C. y Romano, A. M. (2007). La enseñanza de las disciplinas proyectuales: hacia la construcción de una didáctica para la enseñanza superior. Buenos Aire: Nobuko. Morin, E. (2003). Introducción al pensamiento complejo. Barcelona: Gedisa.

Naselli, C. (1997). Bases Epistemológicas del Proceso de Diseño Arquitectónico. Maestría en Diseño. FAUD. Universidad Nacional de San Juan UNSJ. San Juan. Nelson, L. M. (2000). Capítulo 11 "Resolución de Problemas en colaboración" en Reigeluth, Ch. (2000). Diseño de la Instrucción: Teorías y modelos. Un nuevo paradigma de la teoría de la instrucción. Madrid: Aula XXI Santillana. Nonaka, I.T. H. (1999). La organización creadora de conocimiento. México D.F.: Oxford University Press. Piñon, H. (2006). Teoría del Proyecto. Barcelona: UPC. Quintanilla, M. Á. (1991). Tecnología: un enfoque filosófico. Buenos Aires: EUDEBA. Reigeluth, Ch. (2000). Diseño de la Instrucción: Teorías y modelos. Un nuevo paradigma de la teoría de la instrucción. Madrid: Aula XXI Santillana. Romano, A. M. (2015). Conocimiento y practica proyectual. Buenos Aires: Infinito. Sarquis, J. (2003a). "Teoría de la arquitectura, entre la sophia y la episteme" en Sarquis, J. (2003a) (comp.). Teoría de la Arquitectura y Teoría del Proyecto. Buenos Aires: Nobuko. Sarquis, J. (2003b). Itinerarios del Proyecto (Tomo I). Buenos Aires: Nobuko. Sarquis, J. (2004). Itinerarios del Proyecto (Tomo II). Buenos Aires: Nobuko. Schank, R.; Berman, T. y Macpherson, K. (2000). Capítulo 8 "Aprender a través de la práctica" en, Reigeluth, Ch. (2000). Diseño de la Instrucción: Teorías y modelos. Un nuevo paradigma de la teoría de la instrucción. Madrid: Aula XXI Santillana. Schön, D. (1998). La formación de Profesionales reflexivos. Madrid: Paidós. Shapin, S. y Schaffer, S. (2005). El Leviathan y la bomba de vacío. Hobbes y Boyle y la vida experimental. Bernal: Universidad Nacional de Quilmes. Solá-Morales, I. (2003). "Prácticas teóricas, prácticas históricas, prácticas arquitectónicas" en Solá-Morales, I. (2003). Inscripciones. Barcelona: Gustavo Gili. Tishman, S.; Perkins, D. y Jay, E. (1997). Un aula para pensar. Buenos Aires: Editorial Aique. The University of Art and Design (1999). Arteology. Helsinki: UIAH, en http://158.132.155.107/ posh97/private/Arteology/Arteology.pdf Verón, E. (1969). "Ideología y Comunicación de masas: la Semantización de la violencia política" en Verón, E. (Compilador) (1969). Lenguaje y Comunicación visual. Buenos Aires: Nueva Visión. Wasserman, S. (1999). El estudio de casos como método de enseñanza. Buenos Aires: Amorrortu. Wittgenstein, L. (1988). Investigaciones filosóficas. Barcelona: Crítica.

Abstract: The aim of this paper is to reflect on some of the assertions made by Donald Schön about the Project Workshop, questioning his claim about the possibility of learning but not about teaching design. In order to base our position, we will not only characterize Schön's gaze as that of a stranger, but we will also resort to other authoritative voices on the subject, voices that come from both designers and design teachers. In order to broaden our arguments we will also point out what remains invisible to Schön's gaze: the theories of and about Design, theories, worth saying must be taught. And finally we will describe of what Schön is surprised at learning by definition and problem solving, an issue that is presented by many pedagogues as an educational innovation but that in the Design workshop has been done for a very long time ...

Key words: design teaching - teaching tradition - design didactics - project workshop - design theory.

Resumo: O objetivo deste trabalho é refletir sobre algumas afirmações feitas por Donald Schön sobre a Oficina de Projeto, pondo em questão sua afirmação sobre a possibilidade de aprender mas não de ensinar Design. Para fundamentar esta posição se caracterizará a mirada de Schön como se fosse de um estranho e se acudirá a outras vozes autorizadas sobre o tema, provenientes de designers e professores de design. Para ampliar os argumentos se marcará aquilo que permanece invisível á mirada de Schön: as teorias de e sobre o Design, 
teorias que devem ser ensinadas. Por último se descreverá o que a Schön surpreende: a aprendizagem por definição e resolução de problemas, assunto que é apresentado por muitos pedagogos como uma inovação educativa, mas que na oficina de Design se faz há muito tempo.

Palavras chave: ensino do Design - tradições de ensino - didática do design - oficina de design - teoria do design.

Enseñar Diseño: La emergencia de la teoría fue publicado de la página 163 a página185 en Cuadernos del Centro de Estudios de Diseño y Comunicación № 67 